findings are also described well, such as the white matter hyperintensity that can reflect microvascular injuries and thus explain dementia or cognitive impairment. Readers are also presented with the cerebrovascular risk factors for Alzheimer disease, as well as the tantalizing topic of the differential contribution of vascular and degenerative processes to a patient's dementia. Readers will be led to ponder the sensitivity of this topic because of the fuzzy boundary between Alzheimer disease and subcortical vascular dementia. We might refine the PET and SPECT studies introduced in this book for possible use in discriminating or characterizing various conditions.

A few types of receptor PET are introduced, including imaging of the nicotinic acetylcholine receptor system and of inflammation in dementia. Considering the importance of inflammation in neurodegenerative and neuropsychiatric illnesses, the emphasis on neuroinflammation PET of microglial activation is appropriate. However, in another chapter, use of the different name of translocator protein PET/SPECT to describe the same technique for multiple sclerosis is annoying. In contrast, the decision to deal with carotid artery imaging of inflammation and atherosclerosis separately in 2 chapters is appealing. The book covers the most common study for imaging of carotid vulnerability in the clinic- $-{ }^{18} \mathrm{~F}-\mathrm{FDG}$ PET - as well as introducing experimental or preclinical imaging of lipid accumulation, imaging of thrombosis, and angiogenesis imaging related to carotid atherosclerotic plaque. These topics seem sufficient to attract physicians' interest.

The epilepsy studies in this book focus on novel PET ligands showing $\gamma$-aminobutyric acid-ergic transmission, serotonergic or opioid transmission, and P-glycoprotein 1. The listed functional PET studies have interesting findings in disclosing the pathophysiology of epilepsy and promise the future use of PET and SPECT in the development of antiepileptic drugs.

Yu Kyeong Kim
Dong Soo Lee*
*Seoul National University College of Medicine
28 Yungundong Chonnogu
Seoul 110-744, Korea
E-mail: dsl@plaza.snu.ac.kr

Published online Aug. 6, 2015.

DOI: $10.2967 /$ jnumed.115.164590

\section{PET and SPECT of Neurobiological Systems}

R.A.J.O. Diercks, A. Otte, E.F.J. de Vries, and A. van Waarde, eds.

New York, NY: Springer, 2014, 818 pages, $\$ 399.00$

This book contains information mainly on up-to-date PET and SPECT studies of pathologic markers and radiotracers targeting neurotransmitters and their possible clinical uses, as well as detailing the enormous recent progress in molecular neurobiology. Because of the excellent organization of the chapters, neuroscientists will easily locate their systems of interests and physicians will quickly locate upto-date information on molecular imaging of specific brain disorders.

The organization of the book is according to the targeting molecules in neurobiologic systems. The first part presents the basics of PET and SPECT imaging while focusing more on preclinical studies. Starting with animal models of neurologic and psychiatric disorders, the fundamentals of small-animal PET and SPECT systems and protocols are covered. Emphasizing the capabilities of quantitation using PET and SPECT, this part is of the greatest help to readers who deal with animal studies. The last chapter in this part of the book describes the influence of genetic polymorphism on PET and SPECT studies of several neuroreceptor molecules.

The second part covers PET and SPECT imaging of specific systems. Except for Chapter 8, which presents PET tracers for $\beta$-amyloid and other proteinopathies, all the chapters cover specific neuroreceptors, transporters, or neurotransmitters. Each chapter introduces the biologic function of neurotransmitter systems and presents specific radioligands followed by preclinical/clinical studies. Detailed reviews of each system will be useful and attractive to any researcher studying specific systems. To explain why and how several radiotracers have been developed for each system, a portion of most chapters is devoted to a detailed introduction of each system and the related radiopharmaceuticals. For example, the chapter on the endocannabinoid system introduces the physiology of cannabinoid receptors in a concise and easy-to-understand manner and their related neuropsychiatric pathologies. After presenting radioligands for cannabinoid receptor subtypes, the chapter emphasizes current imaging studies and potential applications. Despite the holistic nature of the reviews for each system, the contents of the various chapters do not lose consistency and look well organized.

The chapter on the dopamine system briefly presents the radioligands and imaging studies. Considering that this system has been the most actively studied, we expected a better description. The description of clinical uses and outcomes in movement disordersand the studies and findings on receptor occupancy-are insufficient. One chapter deals with central benzodiazepine receptors. It presents their possible usefulness in chronic cerebral ischemia, skipping other applications such as epilepsy. Other chapters, such as that on nicotinic acetylcholine receptor imaging, present a comprehensive summary of knowledge on previously released radiopharmaceuticals and their image findings, followed by up-to-date disease applications. The chapter on opioid receptor imaging presents the historical development and current wide use of a radioligand.

This book accomplished a job that is never easy. The authors describe most of the preclinical studies and include updated information on the development of radiotracers for every neurobiologic system. They rarely deal with the fundamentals of the kinetics for each tracer or with radiochemistry issues, especially for the neuroreceptors. If readers plan to develop a new tracer to target neurotransmitter systems or to find its application, reference to the relevant chapters will be a helpful starter to acquiring well-rounded knowledge in a specific scientific field within a reasonable time.

The editors have done an excellent job on this entire series of books. We envy their success at benefiting future readers.

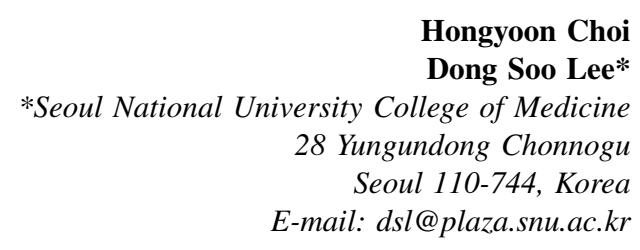

Published online Aug. 20, 2015.

DOI: 10.2967/jnumed.115.164616 